\title{
Philonsorbonne
}

4 | 2010

Année 2009-2010

\section{La relation à autrui chez Bergson}

\section{Éric POMMIER}

\section{(2) OpenEdition}

Journals

Édition électronique

URL : https://journals.openedition.org/philonsorbonne/262

DOI : $10.4000 /$ philonsorbonne.262

ISSN : 2270-7336

\section{Éditeur}

Publications de la Sorbonne

\section{Édition imprimée}

Date de publication : 15 mai 2010

Pagination : 47-67

ISBN : $978-2-85944-647-5$

ISSN : 1255-183X

\section{Référence électronique}

Éric POMMIER, «La relation à autrui chez Bergson », Philonsorbonne [En ligne], 4 | 2010, mis en ligne le 02 février 2013, consulté le 11 juin 2021. URL : http://journals.openedition.org/philonsorbonne/262 ;

DOI : https://doi.org/10.4000/philonsorbonne.262

(C) Tous droits réservés 


\title{
La relation à autrui chez Bergson
}

\author{
Éric Pommier
}

Au cœur d'un moment historique créant les conditions philosophiques qui permettront au problème d'autrui d'occuper une place de toute première importance dans ses approches avant tout phénoménologiques ${ }^{1}$, il semble que Bergson nourrisse une curieuse indifférence à l'égard de cette question. C'est cette indifférence que nous voudrions interroger, afin de savoir si elle est le fait d'une négligence, la reconnaissance implicite de la futilité d'une telle question, ou bien un aveu inconscient: autrui ne peut être qu'un obstacle pour une philosophie d'allure misanthropique, qui ne voit de salut que dans la recherche d'une liberté solitaire. Jusqu'à Matière et mémoire au moins, Bergson s'interroge sur la nature de la liberté et en voit l'essence dans la contraction plus ou moins tendue de notre mémoire personnelle qui trouve à s'incarner, sans se trahir, dans des actes et des œuvres qui s'en imprègnent. En ce sens, autrui apparaît plutôt comme un obstacle à cette ressaisie de soi. Il n'est pas un intermédiaire privilégié permettant la prise de conscience de soi par l'objectivation de mon intériorité. Il est plutôt celui qui s'oppose à cette mise en présence de soi-même, en me contraignant à une communication utile mais superficielle, et superficielle car utile. Dès lors, autrui m'empêche d'avoir accès à la profondeur de mon moi en me maintenant à la surface, au niveau du moi social, du moi commun aux membres d'une société. Il n'y aurait donc de bonheur et de liberté que dans la misanthropie qu'une communication avec autrui, limitée au seul plan de

\footnotetext{
1. C'est dans la cinquième des Méditations cartésiennes que la question de la reconnaissance de l'autre se posera dans toute son acuité. Mais cette question ne pourra se poser qu'au terme d'une évolution de pensée, qui commence dès la première Recherche logique de 1901, avec le problème de la réduction : comment la réduction à la conscience pure et absolue peut-elle rendre compte de la pluralité des consciences absolues?
} 
l'échange nécessaire à la satisfaction des besoins vitaux, masquerait mal. Autrui est donc au fond celui qui parasite le rapport direct que je pourrais avoir avec moi-même, parce qu'il me contraint à développer en moi l'esprit communautaire. En lieu et place d'un homme libre, il y aurait un esprit grégaire. D'autrui, il faut donc se méfier car il ne permet pas l'assomption de la liberté mais favorise l'aliénation, la perte de soi dans l'extériorité.

Mais l'alternative est-elle vraiment entre une profonde liberté solitaire et une sociabilité superficielle, entre un moi profond mais seul et un moi social mais creux ? Pour être libre, faut-il donc être seul, tel un Alceste préférant partir dans le désert plutôt que de conspirer avec la bonne société efficace mais insincère ? Ou bien deux libertés et deux mois profonds peuvent-ils communiquer ? Comme le note de manière lapidaire Vladimir Jankélévitch : «n'y a-t-il pas dans cette immanence même de l'idéal personnel un certain danger de solitude ${ }^{2}$ ?»

Le Rire est le premier ouvrage dans lequel Bergson semble dessiner les lignes d'une éthique. Dans ce texte, Bergson atténue la rigueur avec laquelle il opposait, dès les Données immédiates, le moi social ${ }^{3}$ au moi profond, en montrant comment il y a dans l'échange social plus qu'un échange utilitaire et dans le rapport à soi, fondateur de la liberté, la possibilité d'une ouverture authentique à autrui. Il faudra donc restituer l'échelle souple qui gradue les différents degrés de profondeur dans le rapport à autrui, en montrant à chaque fois la nature d'une telle relation. Ce faisant, on comprendra mieux pourquoi Bergson a dû attendre les Deux sources pour s'intéresser directement à autrui. Si le rapport authentique à autrui doit être un rapport entre deux libertés, alors il fallait savoir en quoi consistait cette liberté et à quelles conditions elle était possible, tâche assignée au moins aux deux premiers ouvrages de Bergson. Ainsi, loin que la liberté soit possible, et même certaine, sous prétexte que la morale se présente comme un fait de la raison, c'est parce que la liberté est un fait que la morale sera possible. C'est en étant libre, en devenant moi-même que je peux me rapporter à autrui afin de le libérer et afin qu'il me libère davantage, par un retour sur soi singulier et réciproque. Il n'y aurait donc d'échange sincère, de communication avec autrui que sous forme d'une morale de la générosité, qui découvre au cœur du soi, dans une philautie renouvelée, un rapport à l'autre. Ce n'est pas en renonçant à soi qu'on découvre autrui ; c'est en s'approfondissant soi-même qu'on peut découvrir la générosité. L'approfondissement intérieur serait bien le moyen d'une extériorisation en direction d'autrui.

\section{Bergson, Paris, PUF, 1959, p. 288.}

3. Chez Bergson la reconnaissance de l'existence d'une autre conscience ne pose pas de difficulté puisqu'au cœur même du moi individuel, il y a toujours déjà la société. C'est ce qu'il thématise au début des Deux sources. En revanche, la reconnaissance d'autrui comme autre conscience singulière et non comme fonction sociale est plus délicate. La communication sociale n'est pas aliénante en elle-même, puisqu'elle est inscrite au cœur du moi. Cependant, elle le devient dès lors qu'elle étouffe le moi profond. 
C'est ce parcours à travers les différents degrés de communication avec autrui que nous souhaiterions faire à présent.

\section{Le lien pragmatique}

La sociabilité est un lien naturel qui me porte vers autrui, mais il repose sur la nécessité de satisfaire des besoins. La communication entre les hommes a donc un but pragmatique, et le langage est constitué de mots renvoyant à des notions générales orientées vers l'action. En ce sens, la misanthropie de l'homme profond serait refus d'accepter la nature agissante de l'homme, refus de satisfaire des besoins sensibles, refus de ne communiquer que sur le registre de l'action ${ }^{4}$. Ce qu'échangent les hommes, ce sont donc des conseils, des recommandations, des commandements en vue d'agir ensemble, afin de pouvoir continuer à vivre. Le lien est donc extérieur. Je ne peux donc pas me conduire en être libre. Parce que je dois satisfaire des besoins sensibles et que je suis dépendant d'autrui pour cela, je ne peux encore me consacrer à la reprise de mon passé en vue de réaliser une action qui me ressemble complètement. Je suis encore asservi à ce qui, en moi, n'est pas moi, à mon être générique. Et je dois utiliser des formes stéréotypées (langage, outils, etc.) pour me conserver. Le rapport à autrui me libère des besoins sensibles, mais sans que cela me permette de réaliser une liberté authentique. La nature du langage tient à cette ambiguïté chez Bergson, des Données immédiates aux Deux sources : d'un côté, le langage 5 rend impersonnel chacun de mes états de conscience en les rendant généraux, homogènes, morcelés, identiques à des choses; de l'autre, ce morcellement rend possible le dialogue entre les hommes. En communiquant les expériences, je suis avec autrui dans l'action, mais je suis seul à être moi dans le retour à mon moi profond, c'est-à-dire à mon moi qui essaie de ressaisir son passé le plus pur, le plus pittoresque, et je ne peux davantage saisir la singularité d'autrui car je ne connais de lui que ce que nous avons en commun : la nécessité d'agir. Autrui n'est encore qu'un moyen pour moi comme je ne suis qu'un moyen pour lui.

4. «On mettrait bien du temps à devenir misanthrope si l'on s'en tenait à l'observation d'autrui. C'est en notant ses propres faiblesses qu'on arrive à plaindre ou à mépriser l'homme» (Deux sources, p. 4). Les faiblesses dont il s'agit ici seraient à la fois le besoin d'agir pour subvenir à ses besoins, le besoin de communiquer avec autrui pour satisfaire ces besoins. Nous approfondirons le sens vrai de la misanthropie.

5. Le langage est dévalorisé dans les Données immédiates et revalorisé dans L'Évolution créatrice : il fait plutôt bien ce pour quoi il est fait puisqu'il permet l'action commune. Il n'est sans doute pas hasardeux de faire remarquer que cette revalorisation du langage social est contemporaine de l'approfondissement de la réflexion esthétique de Bergson. Il existe un langage qui permet de communiquer de manière profonde et assez exacte sa singularité : c'est l'art. En outre la philosophie atteint bien son but puisque Bergson parvient à écrire des livres ! Un langage fluide existe donc. Cf. sur ce point l'article de Bernard Mabille : «Éloge de la fluidité », Les Études philosophiques, octobre-décembre 2001, p. 499- 516. 


\section{Le lien affectif}

Cependant, la société ne réclame pas seulement une contribution pragmatique en vue de satisfaire les besoins vitaux de chacun. Car si c'était là le seul lien social existant, la société dont la fonction est conservatrice se retournerait en son contraire : elle serait destructrice. En effet, il se formerait, au sein de la société, des groupes professionnels avec leur langage propre, mais les individus comme tels ne pourraient plus s'entendre. Ils se formeraient de petites sociétés au sein de la grande, heureuse nécessité si elle n'est pas poussée à son ultime conséquence. En effet, la clôture sur soi de tels groupes entraîne des risques de dissolution.

La société attend donc également une adaptation constante, souple, de chacun à chacun, un effort constamment renouvelé pour que chaque individu soit en harmonie avec autrui. Il ne s'agit plus de vivre simplement, c'est-àdire de survivre. Mais il ne s'agit pas encore de contempler «pour rien, pour le plaisir », de se faire spectateur ou philosophe, dans un renoncement complet à l'unilatéralité de l'action. La société veut vivre, mais aussi vivre bien. Au-delà de la vie mais en deçà de l'art, elle attend que chacun soit à l'écoute de l'autre. C'est là le sens de l'ouvrage sur le rire. Bergson ressaisit une exigence sociale qui est au-delà de la simple nécessité vitale, mais en deçà de la démarche artistique. Le rire est ce geste social qui permet de châtier celui qui s'isole par un comportement trop raide, sans pour autant que ce dernier ne constitue une menace réelle pour la société : la sanction peut donc n'être que légère. Il existe donc, dans la philosophie de Bergson, une sociabilité qui est plus que le respect des codes sociaux ou que l'échange d'informations en vue de l'action, puisqu'elle est souci d'ajustement de l'un à l'autre. Il ne suffit pas en effet de respecter des codes pour être civilisé car à ce compte là, des sauvages ${ }^{6}$ pourraient l'être. Le respect des codes n'a rien à voir avec l'ennoblissement de l'instinct : «Les gens les plus civils ne sont donc pas toujours les plus civilisés $»^{7}$. La société attend de ses membres une communication qui permette de prévenir les dangers propres à la nécessaire division sociale du travail. Celle-ci isole en effet les individus dans des habitudes figées pour les rendre prévisibles et, par conséquent, solidaires les uns des autres ; mais cet isolement se fait au détriment de la compréhension interindividuelle :

En un mot, cette division du travail social, qui resserre l'union des hommes sur tous les points importants en les rendant solidaires les uns des autres, risque de compromettre les relations purement intellectuelles, qui devraient être le luxe et l'agrément de la vie civilisée ${ }^{8}$.

6. Comme le fait remarquer Bergson lui-même, selon une terminologie propre à son époque, dans Mélanges, p. 320.

7. Ibid. C'est le texte fondamental sur « la politesse ».

8. Ibid., p. 321. 
Par conséquent, c'est pour prévenir ce qu'une sociabilité trop pragmatique aurait de dissolvant ${ }^{9}$ pour la société même dont elle permet la naissance, que le lien de type affectif est requis entre les hommes, lien qui, lorsqu'il manque, est signalé par le rire.

Mais en quoi consiste cet ajustement ? Permet-il une communication directe d'une âme à l'autre, d'une mémoire à l'autre ? La relation pure à l'autre qu'on n'a pas trouvée dans le simple échange social en vue de l'action, la trouvera-t-on dans ce que Bergson appelle la politesse de l'âme ?

Ce que le rire châtie, ce sont les mœurs. Lorsqu'un homme, Ménalque ou Alceste $^{10}$, est trop distrait, la société intervient pour corriger cette distraction, interprétée comme étant un début d'isolement. Le châtiment par le rire réintègre alors le distrait, au-delà d'un simple formalisme, dans les bonnes mœurs. Il l'oblige à faire preuve d'une politesse de l'âme, qui requiert de lui attention, tension et élasticité, c'est-à-dire à la fois un regard vigilant sur les sentiments d'autrui et un sens de l'adaptation souple, un appel aux souvenirs communs, ou bien aux souvenirs utiles qui lui permettront de comprendre l'autre ${ }^{11}$. Bergson qualifie cette attitude de sérieuse : le rire rend sérieux. Il pousse à un équilibre de plus en plus délicat des volontés, à un effort constant d'adaptation réciproque.

Mais à quoi tient au fond ce sérieux ? Pour le comprendre, Bergson se demande comment le poète comique fait pour susciter du comique en lieu et place d'un drame. Se demander comment anesthésier notre volonté pour rire de quelqu'un, c'est se demander, en creux, ce qu'il faut pour pouvoir sympathiser avec lui. Savoir en quoi l'homme est comique, c'est comprendre comment il peut être sérieux. En effet, selon Bergson pour rire de quelqu'un il faut faire taire notre sensibilité. Or le sérieux obéit à deux critères : l'attention à soi et l'intérêt pour les actes. C'est parce que l'homme est

9. Le terme n'est pas trop fort: une société qui ne serait que conservatrice, et n'évoluerait plus, ne se conserverait même plus. Dans le dernier chapitre des Deux sources, Bergson montre que la société close est instinctivement portée à faire la guerre. La mécanique prépare la mystique, en nous libérant de nos besoins élémentaires, mais ce développement mécanique doit lui-même être contrôlé par la mystique, afin d'échapper aux tentations guerrières.

10. On voit comment le concept fluide du rire permet de ressaisir les formes extrêmes : depuis le distrait le plus grossier au distrait le plus moral, il y a en commun l'insociabilité. Le rire ne châtie pas l'immoralité mais le manque de souplesse sociale.

11. Cette politesse de l'âme se traduirait sans doute par cet effort de compréhension qu'on manifeste lorsqu'on essaie de se mettre directement dans la pensée d'autrui. Il faut se reporter ici aux pages de Matière et mémoire sur le phénomène d'intellection. Comprendre autrui, c'est aller du sens aux signes et non des signes au sens. On doit aller directement au sens, au mouvement de pensée d'autrui, si on veut le comprendre. Cf. Matière et Mémoire, p. 129. En cela consiste pour Bergson « la faculté de se mettre à la place des autres, de s'intéresser à leurs occupations, de penser de leur pensée, de revivre leur vie en un mot, de s'oublier soi-même» (Mélanges, p. 322). C'est la figure de l'homme du monde qui peut saisir les sentiments et les idées d'autrui de manière singulière, «entrer dans ses vues », comme on dit. Voilà pourquoi une certaine méfiance peut être nourrie à l'égard d'un tel homme : du désir de servir les gens à l'art de se servir d'eux, il n'y a qu'un pas. Mais pour Bergson cette politesse raffinée est avant tout « un désir de plaire », un début de grâce. 
inattentif à soi qu'il ne ressaisit pas son moi profond et laisse un état d'âme parasiter l'ensemble de son âme ${ }^{12}$. Un avare, en effet, n'est pas forcément comique si le poète dramatique sait en restituer la profondeur pathétique ${ }^{13}$. Mais ce qui est sérieux, ce sont aussi les actes. Or ce second critère n'est qu'un prolongement du premier. En effet, un acte n'est sérieux que parce qu'il incarne notre plus ou moins grande liberté. L'action se colore de la profondeur de notre moi, et l'action n'est qu'un prolongement de notre sentiment profond. À l'inverse, le personnage comique est incapable d'action puisqu'il est incapable de profondeur. Il en est réduit aux gestes, c'est-à-dire à des signes dépourvus de profondeur et sans finalité. Notre attention est attirée non sur le tout de la personne, mais sur un aspect superficiel de cette dernière. Il ne faut donc pas s'étonner que le sérieux vienne de notre liberté, c'est-à-dire de notre capacité à ressaisir notre moi profond en sa totalité. Or, c'est à la condition d'un tel sérieux que les conditions d'une sympathie effective sont remplies, qu'un partage est possible, que l'âme peut être dirigée vers autrui pour se mettre à son unisson $^{14}$. Un spectateur ne pourra sympathiser qu'à condition de se rendre attentif aux actes d'un personnage attentif à lui-même. La sociabilité suppose donc avant tout une capacité à se juger soi-même, à se connaître profondément, sans laisser dérober quelque aspect mystérieux. L'inattention à soi est au fond un manque de sociabilité, comme l'insincérité est un manque d'amour, puisque cet intérêt pour soi ne commence que dans le dialogue avec l'autre, l'effort pour lui plaire :

La cause de raideur par excellence, c'est qu'on néglige de regarder autour de soi et surtout en soi : comment modeler sa personne sur celle d'autrui si l'on ne commence par faire connaissance avec les autres et aussi avec soi-même ? ? $^{15}$

Le rire a donc une fonction sociale d'assouplissement ${ }^{16}$ : il est cette mécanique montée par la société en moi qui rit des autres mécaniques. Il vise cependant à nous mettre sur le chemin d'un lien d'une autre nature : la sympathie, l'échange affectif qui commande une attention réciproque des uns à l'égard des autres, en vue d'une meilleure connaissance de soi-même.

12. «Il se forme ici, au sein même du moi fondamental, un moi parasite qui empiétera continuellement sur l'autre. [...] La passion même soudaine, ne présenterait plus le même caractère fatal s'il s'y reflétait, ainsi que dans l'indignation d'Alceste, toute l'histoire de la personne » (Données immédiates, p. 125).

13. « (...) sentiments et affections, désirs et aversions, vices et vertus, tout cela deviendrait une matière à laquelle l'avarice communiquerait un nouveau genre de vie » (Le Rire, p. 108).

14. « (...) comment se découvrirait-il ainsi s'il était capable de se voir et de se juger luimême ? »; et aussi : «Inattention à soi et par conséquent à autrui, voilà ce que nous retrouvons toujours » (Ibid., p. 112).

15. Ibid., p. 112.

16. Le comique s'oppose à l'art - il n'est pas désintéressé - et à la vie - il n'a pas de but simplement pragmatique. 


\section{La communication esthétique ou le refus de la seule « expression »}

Cependant, cette communication affective reste chargée d'ambiguïté. Bergson décrit les conditions d'une entente effective, mais sans en dévoiler la nature. On peut même douter de la profondeur d'un tel échange, en ce qu'aucune indication sur la manière dont deux singularités se rencontrent n'est donnée. Qu'il puisse et doive y avoir rencontre, voilà qui est explicite ; mais la manière dont elle se produit reste implicite.

C'est l'esthétique qui va servir de modèle provisoire pour penser la communication authentique. Grâce à elle, Bergson va penser une relation désintéressée où l'autre comme tel peut se livrer. La difficulté est alors double. D'une part, une relation commune entre deux individualités, dont chacune possède son propre monde, est-elle pensable? D'autre part, en la supposant possible, comment cet échange ne se ferait-il pas au détriment de la société en son ensemble ? En effet, si chacun est rendu à sa nature individuelle dans l'échange avec l'autre, comment la société n'en pâtiraitelle pas? N'y a-t-il pas un risque d'isolement, nuisible à l'intégration sociale ? C'est pour cette raison que Bergson évoque l'art dramatique : il met au jour la profondeur des relations, leur tension intérieure, leur électricité, en suggérant le risque d'une telle sincérité affective : le danger d'une explosion menace lorsque les cœurs se mettent à nu. C'est à cette fin que Bergson convoque l'image du volcan, image qu'il utilisera de nouveau dans l'Energie Spirituelle, à propos des hommes véridiques qui nous remettent en présence de l'élan vital. C'est là rappeler comment la nature sommeille au cour de la société et comment le retour aux sources n'est pas sans risque :

Mais il y a des éruptions volcaniques. Et si la terre était un être vivant, comme le voulait la mythologie, elle aimerait peut-être, tout en se reposant, rêver à ces explosions brusques où tout à coup elle se ressaisit dans ce qu'elle a de plus profond. C'est un plaisir de ce genre que le drame nous procure. Sous la vie tranquille, bourgeoise, que la société et la raison nous ont composée, il va remuer en nous quelque chose qui heureusement n'éclate pas, mais dont il nous fait sentir la tension intérieure. Il donne à la nature sa revanche sur la société ${ }^{17}$.

Cette explosion qui n'aura pas lieu tient à la différence des rythmes qui porteraient les volontés jusqu'au bout de leur effet et engendreraient des tensions aussi inévitables que dangereuses, puisque non réglées par la codification sociale. Il faut donc comprendre comment deux singularités profondes peuvent s'entendre sans menacer pour autant l'existence de la société, soit par leur repli sur elles-mêmes, soit par les tensions qu'elles peuvent engendrer entre elles. Il faut penser un mode de relation sincère qui n'altère pas la nature du lien social, mais qui au contraire le dynamise, afin d'ouvrir la société, sans la détruire pour autant. Il s'agira ultimement de 
définir un lien authentique qui soit libérateur et intégrateur, non destructeur $^{18}$.

Le poète dramatique établit une relation singulière avec le spectateur par la médiation de son œuvre puisqu'il possède l'art de communiquer singulièrement son état d'âme. Shakespeare voit la figure singulière, individuelle d'Othello et c'est elle qu'il communique au spectateur. Mais est-ce seulement l'imaginaire de l'artiste qui nous est livré ou bien la réalité en soi, celle des choses et celle de la conscience ? Pour Bergson, «l'art n'est sûrement qu'une vision plus directe de la réalité ${ }^{19}$. L'art ne nous livre donc pas simplement du beau, mais du vrai, précisément parce qu'il parvient à lever le voile pragmatique qui nous masque la réalité. L'esthétique, en ce sens, est un département de la métaphysique. Mais comment l'état d'âme singulier de l'artiste peut-il être universellement communiqué sans achopper sur le double écueil de l'ineffable et du banal ? Comment le spectateur sait-il qu'il a affaire à l'Othello que Shakespeare a vu ? Comment identifier comme vrai ce qui est unique en son genre ? En réalité, c'est moins le monde créé par l'artiste qui est communiqué, puisque nous ne pouvons pas le voir comme il l'a vu :

Ce que l'artiste a vu, nous ne le reverrons pas sans doute, du moins pas tout à fait de même ${ }^{20}$.

...que l'effort artistique pour voir la réalité comme telle :

L'effort qu'il a fait pour écarter le voile s'impose à notre imitation. Son œuvre est un exemple qui nous sert de leçon. La vérité porte donc en elle une puissance de conviction, de conversion même, qui est la marque à laquelle elle se reconnaitt ${ }^{21}$.

Et encore :

[...] à quel signe reconnaît-on qu'il [le travail artistique] est vrai ? Nous le reconnaissons, je crois, à l'effort même qu'il nous amène à faire sur nous pour voir sincèrement à notre tour. La sincérité est communicative ${ }^{22}$.

Comment expliquer cette impossibilité de l'expression artistique ? Et quelles conséquences peut-on en tirer, du point de vue de la communication avec autrui ? «Les âmes ne sont pas pénétrables les unes aux autres ${ }^{23}$.

18. Et ce seront finalement les figures du héros et du saint - avec les réserves bergsoniennes de la fin des Deux sources sur la possibilité de leur apparition - qui permettront d'ouvrir la société sans en menacer la conservation. La morale ne peut se passer d'un détour par l'esthétique, par la pensée d'une communication singulière qui échappe au double écueil de l'ineffable et de la communication banale.

19. Le Rire, p. 120.

20. Ibid., p. 124.

21. Ibid.

22. Ibid.

23. Ibid., p. 127. 
Bergson affirme ici le principe d'une solitude existentielle (au sens d'une relation-compréhension) : notre moi profond se définit par notre mémoire singulière, qui reste incommunicable, même si elle se matérialise en images qui la manifestent autant qu'elles la voilent ${ }^{24}$. Un obstacle se présente donc à la communication : l'impossibilité de se livrer à autrui comme tel, voire l'impossibilité de se livrer à soi-même, puisque le contact avec le passé pur n'est possible qu'en droit, selon des degrés :

Nous ne pouvons connaître à fond que notre propre cœur - quand nous arrivons à le connaître ${ }^{25}$.

Ce que le poète communique donc, c'est le sentiment d'un effort pour ressaisir la réalité, c'est-à-dire la durée, la mémoire, le sens que les images suggèrent ${ }^{26}:$ l'imagination artistique est en effet une vision non pas directe de la réalité, mais plus directe. La vision directe serait la vision du monde qui dure, la vision d'un artiste total dont chacun des sens verrait les choses dans leur pureté originelle. Ce serait la perception pure qui n'est le fait que d'un fantôme ${ }^{27}$. L'attention à soi, la plongée dans sa mémoire rend possible une vision plus directe de l'existence qui parlera à tout un chacun. Ainsi la prise de conscience de ma temporalité propre et singulière rend possible la rencontre.

Mais alors comment Bergson peut-il parler de sympathie si ce n'est pas dans l'âme d'autrui que je pénètre, et si autrui ne parvient pas à voir ce que

24. Il y a ici une étonnante proximité avec la philosophie d'Emmanuel Lévinas. L'incommunicabilité, sur le mode de l'expression, n'est un manque que si l'on considère qu'il doit y avoir une telle expression. L'amour ne se réduit pas forcément à une relation de compréhension, qui est une façon de nier l'altérité d'autrui en le subsumant sous des déterminations qui sont toujours la manifestation d'un pouvoir, d'une emprise. Chez Bergson comme chez Lévinas, il y a un refus commun de penser la relation à l'autre sur le mode de la connaissance (cf. par exemple E. Lévinas, Le temps et l'autre, Paris, PUF, 1983, p. 83) et la tentative pour penser un mode d'accès à l'autre qui en préserve l'altérité, en vertu d'un principe de pudeur. Mais ils se séparent sur les modalités d'un tel accès. Pour Lévinas, c'est la relation érotique et donc le désir qui permet de penser le rapport au féminin, à l'altérité, en même temps que j'expérimente en moi une pure passivité. Cette passivité est fondamentale car «avec une liberté il ne peut y avoir d'autre relation que celle de la soumission et de l'asservissement » (Le temps et l'autre, p. 80). En revanche, la relation à autrui, chez Bergson, se fait à travers l'émotion et entre deux libertés.

25. Le Rire, p. 127.

26. L'artiste, le créateur est celui qui partant d'un sens, d'un souvenir neuf à communiquer, va le déployer en représentation imagée par l'intermédiaire d'une représentation schématique. Le schéma est d'ailleurs modifié en retour par les images mêmes qu'il évoque. Ainsi opère l'imagination créatrice. Elle comporte une dimension d'effort, car pour "mettre en œuvre » l'impression neuve, il convient de déployer le schéma en images mais aussi de modifier le schéma pour y intégrer les images : il faut donc lutter contre des habitudes anciennes pour en créer des neuves. En cela consiste l'effort (cf. L'Énergie spirituelle, p. 175-179). L'effort ne va donc jamais sans un sentiment de libération, comme si je travaillais à créer une nouvelle habitude qui me permettra de mettre au jour de nouvelles pensées.

27. La perception pure n'est possible qu'en droit : mon inscription corporelle et mon rythme personnel de durée m'interdisent de coïncider complètement avec la matière. 
je vois? En effet, dans le troisième chapitre des Données immédiates, Bergson se demande à quelles conditions réelles Paul peut comprendre Pierre. Or, il y a deux façons d'envisager cette compréhension : statique ou dynamique. Dans la première, j'ai recours à des symboles. Mais alors, je ne peux pas vraiment comprendre Pierre puisque je ne peux savoir quels événements sont déterminants, sont suffisamment intenses pour rendre raison de son comportement. L'intensité des éléments les plus déterminants ne pourra donc être qu'extérieure et quantitative ; mais surtout je ne pourrai mesurer leur caractère déterminant qu'après coup. Je ne comprendrai donc pas ses vécus, puisque c'est seulement en les reconstruisant après coup, que je pourrai expliquer le comportement de Pierre. Mais la compréhension vraie, dynamique, celle par laquelle je saisirais l'intensité qualitative de ses états de conscience, suppose que je connaisse de l'intérieur ses vécus, que je les vive, et donc que je sois Pierre. C'est poser une identification impossible, puisque je n'aurai jamais la même histoire que lui. Il nous semble que Bergson ne fait pas simplement ici la critique du mode de connaissance d'autrui, procédant de l'extérieur, mais qu'il affirme définitivement l'impossibilité de comprendre complètement autrui, sans que cela indique néanmoins un renoncement à le comprendre partiellement, au mieux. Bergson montre que les individualités sont en leur fond incommunicables, et qu'on ne peut en avoir qu'une connaissance approchée. Cela vaut aussi pour la communication médiatisée par l'œuvre artistique :

Ce qui nous intéresse, en effet, dans l'œuvre du poète, c'est la vision de certains états d'âme très profonds ou de certains conflits tout intérieurs. Or, cette vision ne peut pas s'accomplir du dehors. Les âmes ne sont pas pénétrables les unes aux autres. Nous n'apercevons extérieurement que certains signes de la passion. Nous ne les interprétons - défectueusement d'ailleurs que par analogie avec ce que nous avons éprouvé nous-mêmes. Ce que nous éprouvons est donc l'essentiel, et nous ne pouvons connaître à fond que notre propre cœur - quand nous arrivons à le connaître ${ }^{28}$.

En quoi consiste alors cette sincérité si communicative, si elle n'est pas l'expression pure et simple, à l'adresse d'autrui, d'un état d'âme, d'un vécu ?

\section{La limite de la seule relation d'analogie}

Le rapport à autrui est le plus souvent une relation analogique. Je comprends autrui en interprétant, par rapport à ma propre expérience, les signes qu'il m'envoie par ses images, ses mots, ses gestes, son $\operatorname{corps}^{29}$. Je

28. Le Rire, p. 127.

29. Il ne s'agit pas de dire que je remonte des signes extérieurs au sens, mais que je pars du sens que je corrobore par rapport aux signes extérieurs. Cette interprétation n'est jamais pleinement certaine puisque le sens du signe dépend de mon propre vécu. Je ne peux jamais complètement sortir de ma mémoire pure. Cependant, il y a des degrés de certitude. «Entre 
décide d'un sens que je corrobore par rapport aux signes qui me sont envoyés, en faisant un va-et-vient entre sens et signes. On ne connaît donc jamais complètement l'autre en lui-même. En revanche la sympathie qui me porte à une connaissance réellement intérieure de l'autre consiste dans un effort réciproque pour voir les choses en elles-mêmes et sa propre conscience. Ce qui est réciproque, c'est donc un effort de sincérité. Battre au même rythme signifie non pas tant connaître les sentiments de l'autre, son paysage intérieur (même si c'est une médiation nécessaire) que communier dans un effort d'attention aux choses et à soi. Parlant des personnages du poète dramatique, Bergson remarque :

Ce qui nous a intéressés, c'est moins ce qu'on nous a raconté d'autrui que ce qu'on nous a fait entrevoir de nous, tout un monde confus de choses vagues qui auraient voulu être ${ }^{30}$.

Ceci explique un certain privilège de la musique au sein de la hiérarchie des arts proposée par Bergson. La musique en effet, impose à notre attention des rythmes plutôt que des sentiments. C'est la manière ${ }^{31}$ de se rapporter au réel qui peut être partagée. Je ne peux faire comme si j'étais l'autre (car chacun de ses sentiments est vieux de toute son histoire individuelle). Une telle prétention serait manquer de pudeur à l'égard de sa sensibilité en écrasant sa subjectivité par la mienne. Mais je peux en revanche adopter la même tonalité vitale, le même degré de contraction de mémoire pour voir les choses en elles-mêmes. Mon rapport à l'autre est donc non pas d'expression - c'est-à-dire la manifestation extérieure d'un état de conscience intérieure mais de suggestion - c'est-à-dire la communication d'un acte ou d'un rythme de durée ${ }^{32}-$, et il admet des degrés de profondeur. Nous pouvons donc mieux comprendre le sens de la misanthropie : elle manifesterait la contradiction entre le besoin d'une communication authentique et profonde sous forme d'une expression de soi, et le constat qu'une telle expression totale est impossible, le seul mode d'expression efficace étant la relation pragmatique. Le misanthrope refuserait de se contenter d'une relation à l'autre simplement humaine, pragmatique. Mais au lieu de ménager la sensibilité d'autrui dans une posture suggestive, il chercherait plutôt à briser

vous et moi il y a une ressemblance extérieure évidente ; et de cette ressemblance extérieure vous concluez, par analogie, à une similitude interne. Le raisonnement par analogie ne donne jamais, je le veux bien, qu'une probabilité ; mais il y a une foule de cas où cette probabilité est assez haute pour équivaloir pratiquement à la certitude» (L'Énergie spirituelle, p. 6). Bergson retrouve ici une idée présente dans la philosophie de Malebranche : De la recherche de la vérité, III, II, 7, §5. On ne peut en effet connaître autrui que par conjecture.

30. Le Rire, p. 123.

31. Le quod et non le quid, autrement dit.

32. Rappelons que Bergson distingue deux types d'intensité dans les Données immédiates. L'intensité quantitative renvoie au concept de nombre. L'intensité qualitative renvoie à la contraction intérieure de nos différents états d'âme qui s'interpénètrent. Il y a donc différents rythmes de durée, ou différents degrés de profondeur, selon que mes actes traduisent une plus ou moins grande part de mon histoire, selon un degré de personnalisation plus ou moins grand. Il faut renvoyer ici à l'image du cône dans Matière et mémoire (cf. p. 169). 
la solitude dans l'expression : opération impossible puisque la relation doit être d'analogie. Naturellement incompris, Alceste ne peut que manquer le seul rapport possible qui existe entre deux solitudes existentielles: la reconnaissance réciproque de l'effort authentique pour voir les choses en elles-mêmes ou ses états intérieurs selon une profondeur aux degrés variables $^{33}$. Choisir d'entrer dans le rythme de la danse, c'est se faire philanthrope, lorsque le misanthrope s'isole encore davantage en cherchant l'expression de ses sentiments :

En dégageant, en accentuant cette musique, ils [= les compositeurs de musique] l'imposeront à notre attention ; ils feront que nous nous y insérerons involontairement nous-mêmes, comme des passants qui entrent dans une danse. Et par là ils nous amèneront à ébranler aussi, tout au fond de nous, quelque chose qui attendait le moment de vibrer ${ }^{34}$.

Alceste a raison de refuser la conduite simplement sociale de Célimène, mais il a tort de rechercher l'expression d'une vérité. Prisonnier de cette alternative, il ignore son propre rythme et donc celui d'autrui. La coïncidence expressive avec autrui est donc impossible. Lorsque Bergson en parle, c'est d'ailleurs sur le mode conditionnel ${ }^{35}$.

\section{La suggestion et la grâce}

Précisons alors le sens à donner à cette communication suggestive, plutôt qu'expressive. Il ne s'agit pas de renoncer à l'effort d'expression. Je peux comprendre autrui d'autant plus que mon effort d'analogie sera précis. Ce souci d'expression et d'interprétation est par ailleurs la médiation obligée

33. La figure d'Alceste est récurrente sous la plume de Bergson. Dans les Données immédiates, p. 25, Alceste est ce misanthrope superficiel car passionné. Or, note Bergson, les emportements d'Alceste pourraient être sincères, s'ils s'approfondissaient au lieu de ne concerner que la périphérie de sa personne. Derrière cette remarque aux allures d'énigme, Bergson suggère, selon nous, qu'une misanthropie qui s'approfondit se retourne en son contraire : la philanthropie est le constat qu'en lieu et place de l'impossible expression de soi, il y a place pour une communication de la profondeur, sous forme de suggestion. La solitude misanthropique serait un passage nécessaire afin de mieux saisir la nature profonde de la vie, qui est de donner une activité, plutôt que de transmettre un contenu. C'est pourquoi dans les Deux sources, p. 4, la misanthropie est présentée, non comme une haine de l'humanité, mais comme le constat malheureux de sa dépendance pragmatique aux autres, dépendance qu'il faut accepter si l'on veut atteindre un autre degré de communication. En outre, dans Le Rire, p. 105, Bergson prend bien soin de noter qu'Alceste est comique, parce qu'il est associable et bien qu'il soit moral... Il y a manifestement, selon Bergson, dans la figure d'Alceste, les germes de la plus haute moralité : la prise de conscience de sa singularité, si elle ne trouve pas son dernier mot dans la solitude, met sur la voie de la philanthropie : libéré de la banalité, je peux libérer les autres de cette même banalité.

34. Le Rire, p. 120.

35. «Description, histoire et analyse me laissent ici dans le relatif. Seule la coïncidence avec la personne me donnerait l'absolu » (La pensée et le mouvant, p. 179). 
d'une relation à autrui ; car c'est la mémoire comme contenu, comme histoire individuelle qui est livrée dans l'effort d'expression. Mais il s'agit de reconnaître que cet effort d'expression ne peut nous livrer le tout de la personne, et que la relation authentique à autrui n'est pas simplement d'expression. On ne connaîtra jamais, par ce moyen, sa manière particulière d'aimer et de haïr, son style profond. On ne peut donc se contenter d'une relation d'expression. Est-ce alors qu'il faille se porter vers une relation de type hypnotique, où je suis porté magiquement à adopter la volonté d'autrui, à recevoir son influence au point de devenir lui ? Mais alors ce n'est pas l'autre individualité que j'abolirai dans la relation d'analogie expressive, c'est ma propre individualité. Ce n'est plus l'autre qui se perd dans moi, c'est moi qui me perds dans l'autre. Ce ne sont plus des erreurs de la fusion dont je suis victime, mais de celles de l'extase. Il faut donc penser un mode relationnel où chacune des deux singularités est conservée tout en envisageant leur communication, ce mode relationnel étant justement la suggestion.

La relation de suggestion ne me porte pas à cesser d'être moi, mais elle me permet d'être réceptif au rythme d'autrui, et réciproquement. Ce n'est donc pas un contenu de mémoire, mais un degré de contraction par quoi je peux percevoir chez autrui son style (plus ou moins singulier selon le degré de contraction, selon le degré de liberté) qui est communiqué. Je ne saisis certes pas chez lui tel sentiment amoureux, mais sa manière particulière d'aimer. Ce que je saisis c'est le degré personnel de liberté et de personnalité qu'il semble atteindre, c'est le degré de sincérité qu'il semble fournir pour révéler dans son action la profondeur de son moi. C'est avec cela que je suis susceptible de coïncider tout en gardant mon histoire propre. Comprendre autrui n'est pas ressaisir de l'intérieur son histoire personnelle mais se rendre compte :

- Qu'on ne peut jamais avoir totalement accès à cette histoire personnelle. Il y aurait ici un principe fondamental de pudeur chez Bergson. Surtout, «je ne cherche pas à prétendre te connaître». Ce serait un arraisonnement. Et c'est cette pudeur qui permet de ne pas laisser mon ego écraser l'autre et qui permet d'essayer d'utiliser l'analogie sans violence.

- Qu'on peut accéder au rythme de contraction de la mémoire dans un tempo commun qui n'abolit pas les individualités. Simplement, je me mets à l'unisson du degré d'effort fourni par autrui. Je me place au même niveau de tonalité vitale : dans le registre de l'action réfléchie ou de la communication esthétique profonde et sincère.

C'est la raison pour laquelle voir un homme libre pousse à adopter le même rythme de durée que lui et donc à se libérer! Comme le note très justement Vladimir Jankélévitch :

La liberté ne confère pas ceci ou cela, ne fait pas ceci ou cela, mais elle donne envie d'être libre : la généreuse liberté, la liberté héroïque réveille la liberté chez les autres! [...] L'initiative créatrice induit immédiatement, magiquement, télépathiquement de l'un à l'autre les initiatives recréatrices - 
car l'initiative est contagieuse. [...] C'est le temps qui est ici le principe mobilisateur de l'univers inerte. La liberté n'est-elle pas une durée condensée dans l'instant génial ? ${ }^{36}$

Lorsque je suis mis en présence d'un rythme essentiel, d'un rythme qui indique un contact de la personne avec son moi profond, alors je suis invité à me remettre en présence de moi-même, de mon moi profond. C'est cette coïncidence de rythme qui signale une véritable communication, reconnaissance dont l'émotion ${ }^{37}$ est le signal. On voit que la liberté bergsonienne telle qu'elle se rapporte à ma mémoire personnelle n'est pas l'affirmation d'un solipsisme radical. Si Bergson refuse l'idée d'une communication expressive totale, c'est pour mieux dégager un horizon de communication plus essentiel. Si Bergson ne parle jamais thématiquement de l'autre comme tel, c'est qu'autrui est à la fois ce qui requiert une attitude pudique en même temps que suggestive. Autrui est pour Bergson une évidence en même temps qu'une invitation à la discrétion.

Mais comment entre-t-on concrètement dans la danse ? Comment autrui peut-il m'être accessible dans le partage d'un rythme commun ? Disons quelques mots sur le procès de cette identification particulière, et en quoi le concept de rythme est fondamental pour en comprendre la nature. Le modèle esthétique, ici encore, permet de comprendre une telle communication, en même temps qu'il en est une incarnation. C'est la grâce qui permet de saisir au plus près cette entente. Bergson en propose le concept (fluide) dans le premier chapitre des Données immédiates. Le sentiment de la grâce comporte trois éléments qui se fondent les uns dans les autres :

1) C'est une certaine facilité à se mouvoir dont le secret réside dans ce que l'attitude présente semble préformer l'attitude future.

2) Cette facilité entraîne donc un plaisir correspondant au sentiment de pouvoir arrêter la marche du temps puisque l'avenir semble inscrit dans le présent.

3) Cette maîtrise de l'avenir s'intensifie avec le rythme qui semble nous donner le contrôle de l'attitude à venir. Parce que nous pouvons la deviner, nous semblons en être le maître, ce qui rend possible « une espèce de communication ». C'est à ce dernier élément que tient «l'irrésistible attrait de la grâce »: la sympathie physique a une affinité avec la sympathie morale. « Mais la vérité est que nous croyons démêler

36. Op.cit., p. 294.

37. On verra quelle place occupe l'émotion dans les Deux sources : l'émotion est ce que la personnalité morale ou le héros communiquent aux hommes. C'est donc un sentiment esthétique entendu comme épreuve du beau qui nous met en mouvement. Le beau est le désintéressement dans la sphère de la sensibilité. Il est ce rapport à soi désintéressé qui me pousse à être sincère, c'est-à-dire à donner un style à mon existence, en trouvant mes formes propres, en ressaisissant mon passé individuel pour l'incarner dans l'acte ou l'œuvre présente. Cet effort de création de soi par soi, chez le héros ou le saint, est au fond effort artistique, effort du vivant pour se donner une forme individuelle propre. L'émotion dégagée par un tel effort, devant cette beauté, est ce qui me pousse à faire de même. 
dans tout ce qui est très gracieux, en outre de la légèreté qui est signe de mobilité, l'indication d'un mouvement possible vers nous, d'une sympathie virtuelle ou même naissante. C'est cette sympathie mobile, toujours sur le point de se donner, qui est l'essence même de la grâce supérieure $»^{38}$.

Ainsi la grâce est le sentiment éprouvé par une conscience en présence de l'immatérialité qui s'incarne dans la matière ${ }^{39}$, et qui semble s'adresser à elle $^{40}$. Le sentiment esthétique est donc ce qui est suggéré plutôt que causé ${ }^{41}$, ce qui prend la forme de la grâce, c'est-à-dire d'une communication qui essaie de vaincre la résistance de la matière. Comment l'artiste s'y prend-til ? Le rythme est ce qui permet d'hypnotiser le spectateur en le mettant dans l'état de docilité requis pour recevoir la suggestion. Cela vaut pour la musique mais aussi pour la poésie où les images épousent un certain rythme. C'est à l'écoute de ce rythme et de ces images que nous pourrons ressentir l'équivalent émotionnel. Ainsi pouvons-nous voir «avec le poète ${ }^{42}$. Les deux individualités ne s'effacent pas; en revanche le spectateur peut parvenir à éprouver partiellement ce que l'artiste a éprouvé :

(...) et il semble qu'il faudrait revivre la vie de celui qui l'éprouve pour l'embrasser dans sa complexe originalité. Pourtant, l'artiste vise à nous introduire dans cette émotion si riche, si personnelle, si nouvelle, et à nous faire éprouver ce qu'il ne saurait nous faire comprendre ${ }^{43}$.

Parce que le spectateur ne peut vivre de la vie de l'artiste (refus d'une pure coïncidence intérieure) et qu'il ne peut le rejoindre simplement par une connaissance extérieure, par l'expression, il reste une communication visant à me faire adopter le rythme de durée de l'artiste, en créant les conditions favorables à cette adoption :

Il [= l'artiste] fixera donc parmi les manifestations extérieures de son sentiment, celles que notre corps imitera machinalement quoique légèrement,

\section{Données immédiates, p. 10.}

39. «L'immatérialité qui passe ainsi dans la matière est ce qu'on appelle la grâce » (Le Rire, p. 22).

40. Comme le dit de manière suggestive Bento Prado, même si nous n'irions pas jusqu'à envisager une identification totale des contenus de conscience : «Autant dans le cas de la sympathie morale que dans celui de la sympathie physique, ce qui se produit est un processus d'intériorisation, où l' « autre » devient « soi-même », où l'espace « séparateur » est dilué dans une temporalité dans laquelle les parties s'identifient, dans laquelle, enfin la liberté se fait règle ultime, ne rencontrant aucune résistance de la part de la matière : l'esprit de Dieu glissant sur les eaux » (Bento Prado, Présence et champ transcendantal, Hildesheim, Olms, 2002, p. 58).

41. Données immédiates, p. 12. Ce n'est donc pas parce qu'elle n'est pas causalité de l'esprit sur la matière que la suggestion est pour autant de la magie.

42. Données immédiates, p. 11.

43. Ibid., p. 13. 
en les apercevant, de manière à nous replacer tout d'un coup dans l'indéfinissable état psychologique qui les provoqua ${ }^{44}$.

Suggérer, ce sera donc proposer des images qui permettront au corps de prendre l'attitude requise pour diriger l'esprit vers le sentiment unique. Cette imitation du corps sera légère car elle devra être gracieuse, c'est-à-dire orientée vers la pensée de l'artiste et animée par le sentiment à retrouver. On ne retrouvera jamais le sentiment comme tel éprouvé par l'artiste, car ce sentiment est lourd de l'histoire de l'artiste, mais on aura pris l'attitude requise pour le retrouver au mieux, avec sa propre histoire. Ce qui sera de toute façon partagé, ce sera cet effort commun pour atteindre la conscience derrière l'espace :

Ainsi tombera la barrière que le temps et l'espace interposaient entre sa conscience et la nôtre ${ }^{45}$.

Chacun conserve sa durée personnelle, son histoire individuelle, mais le rythme est commun, et je peux essayer de comprendre le sentiment d'autrui à partir des indications qu'il me donne ${ }^{46}$. Si un sentiment esthétique a un caractère gracieux, c'est donc qu'il indique qu'un artiste veut suggérer à un spectateur un sentiment, quelque chose de son moi profond. Cette suggestion sera d'autant plus complète qu'elle se rapprochera de l'émotion, en s'éloignant de la sensation ${ }^{47}$. L'émotion se distingue de la sensation, en ce qu'elle est riche de nombreux états d'âme, qui permettent, plus ou moins, de totaliser l'histoire de l'artiste. C'est parce que l'artiste parvient à nous communiquer ce qu'il y a de plus profond dans sa vie, qu'il pourra nous émouvoir. La grâce est donc cette espèce de communication supérieure qui dépasse l'expression au profit de la communication d'un rythme, d'un degré de profondeur qui pourront être partagés : ce qui compte est moins la mise en commun du même sentiment que la mise en commun du même effort pour voir comme l'artiste a vu. Ce qui compte n'est pas de voir ce qu'il a vu mais, encore une fois, de faire comme il a fait. Suggérer, ce n'est pas délivrer un contenu de conscience, c'est induire un acte, même s'il est vrai que l'induction d'un tel acte se fait à l'occasion et en vue de la communication d'un sentiment. On comprend alors que Bergson oppose le comique au gracieux dans Le Rire puisqu'est comique la raideur d'un corps qui fait chuter l'âme dans le corps. Est comique l'attitude d'un corps qui ne fait signe vers aucun sentiment intérieur et vers personne. La grâce est bien une forme supérieure de communication comme le comique est la forme même d'un être livré à l'isolement et à l'automatisme.

\section{Ibid.}

45. Ibid., p. 14.

46. Pour percevoir cette impression neuve, il faudra remonter de l'image au schéma et atteindre le sentiment. Plus précisément, il faudra avoir une idée du sentiment qui sera corroborée par le jeu des images.

47. Cf. Données immédiates, p. 13. 
La relation à autrui est donc de proximité et de distance : autrui a une mémoire pure personnelle que je ne peux m'assimiler - ce serait vivre sa vie. En ce sens, autrui est à distance. Mais cette distance rend possible autre chose que l'expression par laquelle je ne rencontrerai jamais autrui comme tel mais un masque, un personnage, le héros d'un récit. Elle invite à la proximité de deux consciences, pouvant se rencontrer sur le terrain du rythme partagé, par quoi les actes de mémoire peuvent coïncider dans la recherche commune de la liberté. Une volonté libre s'enrichit de la rencontre d'une autre volonté libre. On voit donc que le rapport à autrui suppose un approfondissement de soi, et que l'approfondissement de soi suppose un rapport à autrui. C'est un cercle dynamique et vertueux que nous propose ici Bergson. C'est une contagion de joie à laquelle il nous invite. Faire de sa vie une œuvre d'art, voilà ce à quoi Bergson nous invite, nous qui sommes des dieux en puissance, sans avoir la frivolité du dandysme ${ }^{48}$ pour autant, puisqu'il s'agit d'une création de soi par soi et non d'un souci extérieur du beau. La politesse que nous nous devons les uns aux autres est bien «la grâce de l'esprit $»^{49}$.

\section{Le retour à la source ${ }^{50}$ ou comment suggérer la générosité}

En montrant que la communication vraie consiste dans une envie commune d'être sincère, nous n'avons pas encore montré quelle était l'origine de cette envie d'être soi ${ }^{51}$. D'où peut venir l'énergie, me permettant de me remettre en présence de moi-même, et d'inviter autrui à la libération ? Quelle est l'origine de l'action morale, de l'action qui conduit à s'intéresser directement à autrui et à agir pour lui ? C'est dans les Deux Sources que Bergson répond à cette question. Il faut dire qu'il a désormais toutes les clefs

48. Le dandy souhaite aussi faire de sa vie une œuvre d'art, comme en témoigne le portrait de Brummell dressé par Barbey d'Aurevilly. Mais cette créativité est moins entreprise de libération individuelle qu'asservissement à la société : en bousculant les normes esthétiques de la société, je reste dépendant d'elle en voulant être reconnu comme me distinguant de la norme sociale. Je dois me déterminer par rapport à une norme commune précisément pour pouvoir la rejeter. C'est là toute la contradiction du dandy.

49. Mélanges, p. 324 : «Comme la grâce, elle [la politesse] éveille l'idée d'une souplesse sans bornes ; comme la grâce, elle fait courir entre les âmes une sympathie mobile et légère ; comme la grâce enfin, elle nous transporte de ce monde où la parole est rivée à l'action, et l'action elle-même à l'intérêt, dans un autre, tout idéal, où paroles et mouvements s'affranchissent de leur utilité et n'ont plus d'autre objet que de plaire ».

50. Nous n'aborderons pas la question du statut ultime de la source dernière de l'être : Dieu ou élan vital. Nous utiliserons par commodité l'un ou l'autre de ces termes, en pensant à une même chose : la vie est le principe dernier de l'univers pour Bergson.

51. La communication esthétique ne permet pas encore de penser complètement le rapport à l'autre, puisqu'elle en reste au plan des images. Il faut donc aborder à présent Les Deux Sources pour penser un rapport direct, et non plus médié par l'œuvre d'art, à l'autre. En outre, l'artiste n'entre pas encore en contact avec la source même de la vie, seule dispensatrice de générosité. 
en main pour la résoudre. Ses deux premiers ouvrages lui ont permis de saisir la nature de la liberté. L'Évolution créatrice lui a permis de trouver un mode d'accès gnoséologique à l'origine radicale de toutes choses en réinterprétant de manière originale cette question : c'est par la psychologie que je peux comprendre la cosmologie, car c'est dans le rapport à soi que je peux intuitionner, brièvement et partiellement, la manière dont l'univers se crée à tout moment. Je fais l'expérience de ce qu'est l'élan vital, de la source dernière de toutes choses, de l'homme, et donc de moi aussi, en ce que je participe de cet univers de manière active.

Dès lors Bergson peut réfuter une morale qui verrait dans la raison en lutte avec notre égoïsme naturel la source de l'action désintéressée. La seule façon de penser une action morale effective (et qui ne soit pas un pur et simple automatisme social, mais une action qui intéresse le tout de l'humanité) consiste plutôt à se retourner sur soi pour expérimenter en nous la présence de la source, de l'élan vital qui agit encore et à tout moment. C'est parce que notre mémoire personnelle est solidaire d'une mémoire universelle plus vaste que nous pouvons reprendre contact avec ce qui n'a jamais été oublié, avec ce qui est cosmologiquement mémoire ${ }^{52}$. Or cette expérience de la participation de soi à un tout qui nous dépasse et auquel nous participons est l'expérience même de notre solidarité à l'effort universel de création. Nous ne sommes pas que des êtres d'instinct (instinct certes sympathique dans le cas du sphex de L'Évolution créatrice mais sympathie égoïste, intéressée, inconsciente) ni d'intelligence (qui nous porte plutôt vers l'égoïsme que vers la solidarité sociale comme le rappelle les Deux sources) mais des êtres d'intuition. Pour avoir une connaissance précise de ce que nous sommes, nous pouvons retenir de l'instinct la connaissance intérieure de son objet, et de l'intelligence sa connaissance consciente et réfléchie, en abandonnant ce que le premier a d'inconscient et le second de statique. Je suis donc mis en présence de l'origine de l'être sur un mode métaphysique. Il ne s'agit pas de savoir quelle est la raison sociale, psychologique de mon existence mais bien de connaître la raison de toutes ces raisons. Et la raison dernière se fait dans l'épreuve d'une solidarité cosmologique avec tout ce qui est, selon des degrés de durée différents. C'est dans une conscience supérieure que je réalise que le fond de la vie est généreux. L'Évolution créatrice était déjà sur une telle piste :

Cet amour, où quelques-uns ont vu le grand mystère de la vie, nous en livrerait peut-être le secret. Il nous montre chaque génération penchée sur celle qui la suivra. Il nous laisse entrevoir que l'être vivant est surtout un lieu de passage, et que l'essentiel de la vie tient dans le mouvement qui la transmet ${ }^{53}$.

52. On ne comprendrait pas qu'il soit possible de reprendre contact avec l'origine de la vie, si l'univers n'était pas lui-même une mémoire, entendue comme « coexistence virtuelle » selon la formule de Gilles Deleuze. Cf. Le bergsonisme, Paris, PUF, 1966, chap. 3, p. 45-70.

53. L'Évolution créatrice, p. 129. 
Le secret, ou le principe dynamique de la vie, serait l'amour, ou encore la générosité. La générosité vient donc s'opposer à l'égoïsme de celui qui ne pense qu'à soi, mais dérive pourtant de l'attention à soi, seule capable de nous amener à comprendre que notre direction naturelle est d'aller vers autrui, sur un mode charitable. À la fin de son hommage à Ravaisson, Bergson rappelle que le mobile naturel à l'homme est non pas l'égoïsme mais la générosité.

Or cette générosité ne peut se développer qu'au contact des grandes personnalités sincères qui ont fait exemplairement cette expérience de l'origine. Tel est le cas du héros et du saint (et aussi des grandes personnalités morales). Chacun dans son registre, celui de l'action, celui de la parole, ils laissent entrevoir quelque chose de l'exigence de création qui est le sens même de la vie. Ils nous invitent à notre tour à revenir en nous, à découvrir notre liberté intérieure, à nous rendre généreux. Cette force morale qu'ils communiquent emprunte la voie de la sensibilité. L'homme véridique émeut d'une émotion créatrice. En effet, cette émotion seule, qui est «à la fois la curiosité, le désir, et la joie anticipée de résoudre un problème déterminé », nous remet en présence du tout de notre âme. Elle n'est pas infra-intellectuelle mais supra-intellectuelle. Ainsi elle ne dérive pas d'une représentation de l'intelligence ni n'est synonyme d'un désir qui resterait à la périphérie de notre âme, mais est source de pensées nouvelles et motivantes. Car, contrairement à la conception sartrienne de l'émotion, celle-ci n'est pas l'attitude passive d'un être rendu incapable de transformer techniquement le monde et réduit à le transformer magiquement, mais l'attitude active d'un être qui trouve des forces et des idées nouvelles pour parvenir à incarner son action dans le monde. La source de notre élan est donc bien cette pleine ressaisie de soi qui engendre une insatisfaction motivante afin d'agir. Ce n'est pas du côté du manque inhérent au désir que je trouve l'énergie d'agir moralement, mais dans la plénitude stimulante de l'émotion qui me pousse à insérer de la nouveauté dans le monde.

Mais comment cette générosité pourrait-elle se manifester en moi si elle n'y était pas déjà ? Ce qu'expérimente de manière explicite le saint ${ }^{54}$, c'est au cœur de la vie un principe de générosité, une libéralité, quelque chose comme un principe qui se donne, dira le texte sur Ravaisson. C'est parce que la vie est l'amour même, le don de soi, l'abandon que l'homme véridique est la grâce même. Il est celui qui, dans ses mots et dans ses actes, témoigne de la libéralité propre à la vie, lorsqu'elle se ressaisit dans ce qu'elle a de plus propre :

La question est alors de savoir en quoi consiste la grâce. Mais ce problème est plus aisé à résoudre, car dans tout ce qui est gracieux nous voyons, nous sentons, nous devinons une espèce d'abandon et comme une condescendance. Ainsi, pour celui qui contemple l'univers avec des yeux d'artiste, c'est la grâce qui se lit à travers la beauté, et c'est la bonté qui transparaît sous la grâce. Toute

54. À la différence du héros qui témoigne par l'action, mais pas par la parole. 
chose manifeste, dans le mouvement que sa forme enregistre, la générosité infinie d'un principe qui se donne. Et ce n'est pas à tort qu'on appelle du même nom le charme qu'on voit au mouvement et l'acte de libéralité qui est caractéristique de la bonté divine ${ }^{55}$.

Ainsi ce don du saint consiste à donner non pas quelque chose, mais une activité $^{56}$. Le don est un don d'action qu'il puise au cœur même de Dieu. C'est à partir de la relation privilégiée - permise par l'intuition mystique entre Dieu et lui, que le saint peut expérimenter directement l'énergie créatrice comme amour. Car telle est la raison pour laquelle le don du saint peut être reçu. En effet, comment communiquer à autrui cette activité autrement qu'en le mettant dans une attitude de passivité, en risquant de l'assujettir, par influence ou prise de contrôle ? Comment l'influence peutelle être autre chose qu'un arraisonnement? Comment l'exercice d'une volonté libre peut-elle agir autrement que par la réduction d'autrui à l'état de chose passive ? C'est par la suggestion que ce modèle qu'est le saint va éveiller en moi une envie propre d'agir. C'est parce que je reconnais en lui une source d'activité personnelle que je vais agir à mon tour. L'énergie qu'il communique est donc une envie de donner en créant. L'exigence de création est au fond le don du don, le don de générosité. Le Saint donne à l'autre charitablement comme Dieu a créé avec charité. C'est à l'autre comme fin que s'adresse ce don puisque le Saint donne avec grâce, généreusement. En un mot, les grandes personnalités sincères font l'épreuve, au cœur d'ellesmêmes et de l'être, d'un principe de charité ou de générosité qui consiste dans un don d'activité, de création. Je suis remis en présence de moi-même lorsque je réapprends à donner à autrui la possibilité de donner, en donnant moi-même sous forme de création de soi par soi. Le saint me rappelle à ma vocation d'homme généreux, qui peut donner librement, c'est-à-dire qui peut donner la liberté. Devenir modèle de générosité, telle est l'ambition d'une relation duelle réellement libératrice.

Il existe donc bien une relation authentique entre les hommes. C'est celle qui rend possible le retour à l'origine, à la source, de laquelle on peut tirer l'envie et l'énergie d'être sincère. La communication libre n'est pas celle où un homme tente d'agir sur un autre homme, de manière directe ou bien indirecte, selon la définition foucaldienne du pouvoir, compris comme « action sur des actions », mais bien une coaction où l'un libère l'autre qui le libère en retour un peu plus. L'homme libre redonne confiance en soi en

\section{La Pensée et le Mouvant, p. 280.}

56. Un rapprochement avec Fichte, dont on sait qu'il a été l'objet de cours professés par Bergson à l'École Normale Supérieure, serait ici fructueux. Fichte aussi donne les conditions pour penser une relation entre deux libertés, qui se donnent réciproquement de l'activité. Cf. J.G. Fichte, La destination du savant, Paris, Vrin, 1994, p. 52-54. Et par exemple : « Nous pourrions donc aussi bien dire : perfectionnement commun, perfectionnement de nous-mêmes par l'influence librement consentie des autres sur nous : et perfectionnement des autres par influence en retour sur eux en tant qu'êtres libres, voilà notre destination dans la société » $(L a$ destination du savant, p. 53). 
calmant la peur inhérente à l'envie d'être soi. C'est alors d'un cœur joyeux puisque remis en présence de l'exigence de création - que nous pouvons cultiver «l'élément tragique de notre personnalité » et chanter l'avenir. Il se pourrait d'ailleurs que Dieu lui-même ait besoin que les hommes accomplissent leur vocation de créateurs pour être aimé, et peut-être aussi fortifié ${ }^{57}$.

La relation avec autrui connaît des degrés de profondeur, selon le degré de libération réciproque qu'elle rend possible, depuis la relation de nature pragmatique qui met en rapport deux mois sociaux, jusqu'à la communication suggestive entre deux individualités qui ressaisissent en elles-mêmes le courant même de la vie propre à alimenter leur créativité personnelle. C'est donc l'émotion ressentie pour l'autre qui m'invite à me remettre en présence de mon dynamisme intérieur, afin que, moi aussi, je puisse expérimenter l'envie d'être généreux. C'est pour l'autre et grâce à l'autre que je me libère. La découverte de ma liberté est toujours polarisée par la présence d'autrui. La générosité n'est pas exclusive de l'attention profonde à soi. Celle-ci serait bien plutôt la condition de celle-là.

57. «Par le fait, les mystiques sont unanimes à témoigner que Dieu a besoin de nous, comme nous avons besoin de Dieu. Pourquoi aurait-il besoin de nous, sinon pour nous aimer?» (Deux sources, p. 270). 